\title{
Alcalosis metabólica en fibrosis quística del páncreas
}

\author{
Dru. Teresa Param S. ${ }^{1}$; Dr. Djego Aldunate $\mathrm{G}_{+}{ }^{2}$
}

Metabolic alkalosis in cystic fibrosis

A 3 month old male infants with two eaflier episodes of respitatory infection presented with respiratory symptoms, cianosis, hyponatremia, hypokalemia, hypochloremia, metabolic alkalosis, and normal renal function tests. Quantitative determinations of sweat electrolytes in three samples showed elevated $\mathrm{Na}^{+}(\bar{x} 62.6 \mathrm{mEq} / \mathrm{L})$ and $\mathrm{Cl}^{-}(83.9 \mathrm{mEq} / \mathrm{L})$ concentrations, together with high plasma renin activity $(30 \mathrm{ng} / \mathrm{mL} \times \mathrm{h}$, normal: $1-2.5 \mathrm{ng} / \mathrm{mL} \times \mathrm{h})$. Excesive $\mathrm{Na}^{+}$loses and depletion probably explain high renin activity, aldosterone secretion and the occurrence of metabolic alkalosis in this patient. Cystic fibrosis must be included in the differential diagnosis in children with unexplained metabolic alkalosis and recurrent respiratory symptoms.

(Key words: cystic fibrosis, metabolic alkalosis.)

La fibrosis quistica del páncreas es una enfermedad hereditaria que compromete principalmente a niốos, adolescentes y adultos jóvenes y que se caracteriza por un trastorno funcional generalizado de las glándulas exocrinas.

La presencia de enfermedad pulmonar crónica. deficiencia pancreática y concentraciones anormalmente altas de electrólitos en el sudor constituyen la clásica tríada de presentación ${ }^{1}$, aunque se sabe que no siempre es afectado el páncreas, el compromiso respiratorio está pre. sente en la gran mayoría de los pacientes y es causa importante de muerte ${ }^{2}$.

Mucho menos frecuente es la presencia de otro tipo de alteraciones, como por ejemplo del equilibrio ácido-base y electrolítico. En estos enfermos se produce una alcalosis metabólica y concentraciones bajas de electrólitos sanguj. neos ${ }^{3}$, lo que puede inducir al planteamiento de diversos diagnósticos, si es que no se tiene el antecedente de la existencia de fibrosis quística en un determinado paciente.

Sc describe a continuación et caso de un lactante con alcalosis metabólica hiponatrémica, hipokalémica e hipoclorémica y en el cual se comprobó el diagnóstico de fibrosis quística del páncreas.

1. Servicio de Pediatria Hospíal Militar y Departamento de Medicina Experimental, Div. Ciencias Médicas Norte, Facultad de Medicina, Universidad de Chile.

2. Servicio de Pediatría Hospital Militas.

\section{CASO CLINICO}

Paciente de sexo masculino. Eimbarazo y parto normales, peso de nacimiento $4.080 \mathrm{~g}$, cuenta de apgar 9 , uno y cinco minutos despućs de nacer. A los 45 días fue hospitalizado por bronconeumonía $y$ atelectasia izquierda que se habían resuelto clínica y radiológicamente 7 días después. Padres y 2 hermanos sanos.

Ingresó a los 3 meses de edad por dificultad respiratoria moderada, cianosis distal leve, deshidratación moderada, frecucncia cardíaca 140 pos minuto, frecuencia respiratoria 40 por minuto, temperatura rectal $37,1^{\circ} \mathrm{C}$, estertores pulmonares difusos. En la radiografía dc tórax se observan imágenes intersticiales. Los estudios de gases arteriales y electrolitos en sangre y orina (tabla 1) mostraron alcalosis metabólica, hiponatremia e hipokalemia con excreciones bajas de sodio y cloro y clevadas de potasio. La función renal era normal.

Manejado inicialmente con solucioncs endovenosas de electrolitos, mostró una respuesta favorable, pero durante la hospitalización sufrió un nuevo cpisodio de bronconeumonía con múltiples atelectasias sogmentatias que también respondió satisfactoriamente al tratamiento con penicilina y amikacina.

Ante la ausencia de historia clíniea sugerente de pérdidas digestivas y la presencia de dos episodios de neumopatía dguda, el primero a los 45 dias, se midieron electrolitos en sudor: la eliminación del cloro y sodio cstaba francamente aumentada en tres muestras (tabla 2). No se demostró compromiso digestivo. La actividad de renina en el plasma era de $30 \mathrm{ng} / \mathrm{mL} \times \mathrm{h}(\mathrm{n}=1$ a 2.5 $\mathrm{ng} / \mathrm{mL} \times \mathrm{h}$ sin estimulación en decúbito horizontal).

Se dio de alta en buenas condiciones a los 22 dias de hospitalización.

Las mediciones de $\mathrm{pH}, \mathrm{PCO}_{2}, \mathrm{PO}_{2}$ se realizaron en un analizador de gases Radiometer, modelo BGA3; el sodio $y$ potasio en plasma $y$ orina por fotometría de llama y cl cloro por titulación. La actiuidad de renina en plasma se midió por radioinmunoanálisis, como con- 
Tabla 1

Parámetros ácidos básicos y electrolitos durante los primeros 5 đías de evolución

\begin{tabular}{lcccccc}
\hline & & 10 & 20 & 30 & 40 & 50 \\
\hline & & & & & & \\
$\mathrm{FiO}_{2}$ & 0,5 & 0,35 & 0,42 & 0,40 & 0,40 & 0,40 \\
$\mathrm{pH}$ & 7,54 & 7,66 & 7,52 & 7,48 & 7,47 & 7,41 \\
$\mathrm{PaO}_{2}$ & 101 & 68 & 90 & 57 & 84 & 92 \\
$\mathrm{PaCO}_{2}$ & 59 & 52 & 49 & 46 & 33 & 40 \\
$\mathrm{BE}$ & 23 & +20 & $+14,7$ & $+9,2$ & +1 & +1 \\
$\mathrm{Na}^{+} \mathrm{p}(\mathrm{mEq} / \mathrm{L})$ & 122 & 127 & 133 & 134 & 132 & 142 \\
$\mathrm{Cl}^{-} \mathrm{p}(\mathrm{mEq} / \mathrm{L})$ & 60 & 75 & 87 & 89 & 100 & 105 \\
$\mathrm{~K}^{+} \mathrm{p}(\mathrm{mEq} / \mathrm{L})$ & 1,7 & 1,9 & 3,3 & 3,3 & 4,5 & 4,6 \\
$\mathrm{Na}^{+} \mathrm{u}(\mathrm{mEq} / 24 \mathrm{~h})$ & $5 *$ & 5,6 & 5,2 & 4,3 & 7,0 & 13 \\
$\mathrm{Cl}^{-} \mathrm{u}(\mathrm{mEq} / 24 \mathrm{~h})$ & $3 *$ & 3,5 & 4,4 & 6,2 & 9,0 & 9 \\
$\mathrm{~K}^{+} \mathrm{u}(\mathrm{mEq} / 24 \mathrm{~h})$ & $40 *$ & 6 & 8,2 & 21 & 17,0 & 8 \\
& & 1 & 2 & 3 & 4 & 5 dias \\
\hline
\end{tabular}

$\mathrm{p}=$ plasma; $\mathrm{u}=$ orina

* Muestra aislada $(\mathrm{mEq} / \mathrm{L})$.

Tabla 2

Resultados electrolitos en sudor

\begin{tabular}{lccc}
\hline Muestra & 1 & 2 & 3 \\
\hline $\mathrm{Na}(\mathrm{mEq} / \mathrm{L})$ & 52,3 & 74,0 & 61,3 \\
$\mathrm{Cl}(\mathrm{mEq} / \mathrm{L})$ & 80,4 & 92,0 & 79,5 \\
\hline
\end{tabular}

centración de angiotensina I, genezada en una hora de incubación (Kit New England Nuclear). Los electrolitos en sudor se midieron siguiendo el método clásico de estimulación por iontoforesis con pilocarpina y posteriormente cuantificados por fotometr ía de llama.

\section{DISCUSION}

Se destaca en este paciente la severa alcalosis metabólica con concentraciones bajas de electrolitos en sangre. En los niños la alcalosis metabólica es poco común: puede observarse en pacientes con estenosis pilórica, vómitos persistentes, drenaje de contenido gástrico con inadecuado reemplazo de líquidos y electrolitos, ingestión de alcalis estados asociados a hipoka. lemia (hiperaldosteronismo) y en el síndrome de Bartter. Se ha descrito alcalosis metabólica en la fibrosis quistica del páncreas ${ }^{3-6}$. Aunque no se conoce su real prevalencia y tampoco está clara su fisiopatologia, se podría postular lo siguiente: las glándulas sudoriparas de pacientes con fibrosis quistica secretan grandes cantidades de $\mathrm{Na}$, $\mathrm{K}$, Cl y agua por las secreciones y continúan eliminando estos electrolitos en forma excesiva por el sudor a pesar de un balance negativo especialmente de sodio. Esto prođuciría contracción de volumen extracelular (hipovolemia), que induce disminución de la velocidad de filtración glomerular, de manera que aumenta la reabsorción de sodio, cloro, bicarbonato y agua en el túbulo proximal. Además se estimularía el sisterna renina-angiotensina-aldosterona ${ }^{7}$, to que conduce a reabsorción importante de $\mathrm{Na}^{+}$, excreción de protones y/o potasio, agravándose la alcalosis metabólica y la hipokalemia ya existentes (figura 1) ), $^{\mathbf{9}}$.

Está descrito que existe aumento de la reabsorción de $\mathrm{Na}$ en el túbulo proximal renal, con disminución de la liberación de $\mathrm{Na}^{+}$en los segmentos más distales, lo que explica el ahorro de sodio ${ }^{10}$.

Por otro lado, durante períodos de calor intenso un niño con fibrosis quistica puede eliminar más de $80 \mathrm{mEq}$ de sodio, $100 \mathrm{mEq}$ de cloro y $90 \mathrm{mEq}$ de potasio por día mediante el sudor, dependiendo de la superficie corporal y velocidad de sudoración; además hay grandes 


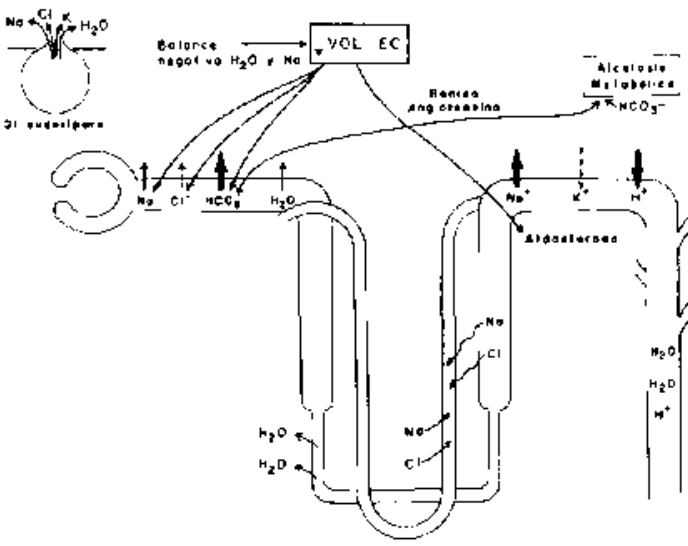

Figura 1. Fisiopatología alcalosis metabólica en fibrosis quística.

pérdidas de líquido, pudiendo ocurrir hipovolemia, que es agravada más aun por la depleción salina. Se recomienda en estos casos el aporte extra de sal ${ }^{11}$.

Este paciente ingresó en época calurosa y con cierto grado de deshidratación, lo que Llevó a realizar como primeras medidas de tratamiento la reposición de agua y electrolitos.

Cuando existe hipokalemia, el balance intracelular de $\mathrm{K}$ es negativo, to que significa entrada de $\mathrm{Na}$ y $\mathrm{H}$ a las células para reemplazar el déficit de $K$, agravándose así la alcalosis metabólica ${ }^{4}, 12$. Además, la hipokalemia mantenida produce una pérdida temporal de la capacidad de concentración renal, con perpetuación del trastomo metabólico (nefropatía kaliopénica) ${ }^{4}$.

El hallazgo de alcalosis metabólica sin historia de vómitos sugiere el diagnóstico de hiperaldosteronismo, ya sea primario o secundario. La aldosterona es el principal mineralacorticoide que promueve el transporte de $\mathrm{Na}^{+}, \mathrm{K}^{+}$e $\mathrm{H}$ por las células del túbulo renal y es de gran importancia en el control normal de líquidos y electrólitos. La secreción fisiológiça de aldosterona está controlada por el volumen de líquido extracelular, angiotensina II, ACTH y niveles de potasio.

La exposición al calor y la depleción de sodio aumentan la secreción de aldosterona, efecto atribuido en parte a la estimulación de la secreción de renina y, asi, de angiotensina.

Se estudió el sistema renina-angiotensina-ałdosterona en pacientes con fibrosis quística y se observó que efectivamente existe hiperaldosteronismo secundario a liberación de renina, probablemente como resultado de una adaptación a excesivas pérdidas de sodio por el sudor, con la consecuente contracción de volumen extracelular. Esto explica la hiperreninemia observada en este niño. El hiperaldosteronismo aumenta las pérdidas de $\mathrm{K}$ e $\mathrm{H}$ por el riñón, contribuyendo a perpetuar la alcalosis metabólica e hipokalemia ${ }^{\top}$.

Otro de los diagnósticos planteados durante los primeros dias de evolución fue el sindrome de Bartter, debido a la excreción urinaria aumentada de potasio con alcalosis metabólica, hipokalemia y presión arterial normal. En eșta afección existe pérdida obligada de cloro $^{13}$. El diagnóstico se confirma estudiando la reabsorción fraccional de cloro en condiciones de sobrehidratación. Los sujetos portadores de sindrome de Bartter presentan valores inferiores a $65 \%$, y los sujetos normales, como los portadores de hipokalemia de cualquier otra etiología, muestran cifras superiores a $80 \%$.

En la mayoría de los casos basta medir el cloro en orina de 24 horas; valores bajos $(<5$ $\mathrm{mEq} \times 24$ horas) sugieren un mecanismo extra. renal como causa del trastorno y valores altos $(<10 \mathrm{mEq} \times 24$ horas) orientan hacia una causa renal. En este paciente se constató que existja ahorro de cloro por el riñón.

Finalmente, el diagnóstico de fibrosis quística del páncreas șe confirmó mediante medición de electrolitos en el sudor ${ }^{4}$. Esta prueba es altamente sensible y especifica, ya que más del $98 \%$ de los pacientes con fibrosis quistica tienen concentraciones de cloro entre 60 y 130 $\mathrm{mEq} / \mathrm{L}^{15}$. Debido a la importancia de esta medición, ella debe realizarse en laboratorios especializados, teniendo presente, además, que valores elevados pueden encontrarse en otras enfermedades ${ }^{15,16}$.

Es muy importante hacer precozmente el diagnóstico de fibrosis quistica, debiendo sospecharse en todos los lactantes o nintos con síntomas recurrentes o crónicos del aparato respiratorio, en recién nacidos con jleo meconial, en niños con síndrome de malabsorción, en pacientes con alcalosis metabólica asociada a concentraciones bajas de electrolitos plasmáticos, sin deshidratación ni evidencias de pérdidas digestivas. E] diagnóstico oportuno de la fíbrosis quística permite mejorar la calidad de vida y aumentar la sobrevida de los niños afectados.

\section{RESUMEN}

Un varón que había sufrido bronconeumonía 45 días después de nacer, ingresó al hospital a los 
3 meses de edad por dificultad respiratoria, cianosis, deshidratación moderada, alcalosis metabólica, hiponatremia, hipocloremia, hipokalemia, excreción urinaria reducida de sodio y cloro y elevada de potasjo. Sus funciones renales eran normales y cn las radiografías de tórax se obser. yaba evidencia de imágenes intersticiales de condensación y múltiples atelectasias segmen. tarias. La actividad de renina plasmática era de $30 \mathrm{ng} / \mathrm{mL} \times \mathrm{h}(\mathrm{n}=1$ a $2,5 \mathrm{ng} / \mathrm{mL} \times \mathrm{h})$ y la eliminación de cloro $y$ sodio en el sudor estaban aumentadas a $\bar{x} 62,6$ y $\bar{x} 83,9 \mathrm{mEq} / \mathrm{L}$, respectivamente, respaldando el diagnóstico de fibrosis quística. La alcalosis metabólica es poco común. En ausencia de causas iatrogenicas debe sugerir hiperaldosteronismo, síndrome de Bartter y fibrosis quistica.

\section{REFERENCIAS}

1 Vivanco $G_{1}$ Cuellor A, Antelo O, Ceruti E.: Fibrosis quística del páncreas. Rev Chil Pediatr 1979; 50: 6-16.

2. Mischler E.: Trcatment of pulmonary disease in cystic fibrosis. Seminars in Respiratory Medicine 1985; 6: 271-283.

3. Davis P.: Miscellaneus complications of cystio fibrosis. Serminars in Respiratory Medicine 1985; 6 : 319-320.

4. Beckerman R.: Taussig L.: Hypoelectrolitemia and metabolic alkalosis in infants with cystic fibrosis. Pediatrics 1979; 63: 580-583.
5. Gottlieb $R$ : : Metabolic alkalosis in cystic fibrosis. J Pediatr 1971; 79: 930-936.

6. Saein C., Rodriguez J., Paris E., Fuentes A. Le. telier C: Alcalosis metabólica Jipokalćnica: importancia del cloro. Rey Chil Pediatr 1982; 53: 233-236

7. Simopoulos A. P., Lapey A., Boat T. Di Sant'Agnese and Bartter $F_{\text {.: }}$ The renin-angiotensin-aldosterone system in patients with cystic fibrosis of pancreas. Pediatr Res 1971: 5: 626-631.

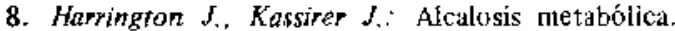
En: Cohen y Kassirer J. eds. El equilitrio ácidobásico y sus trastomos. Barcelona. Salvat 1985.

9. Kaehny H.. Gahow P.: Patogenia y mancjo de la acidosis y la alcalosis metabólicas. En: Schrict R. Trastornos renales y electrolíticos. Barcelona. Salvat 1984.

10. Robson A., Tateishi S., Ingelfinger J.R., Stro. minger $D$., Klath $S$. Renal function in patients with cystic fibrosis. J Pediatr 1971:79: 42-50.

11. Rendle-Short $J$.: Fibrocystic discase of the pantreas presenting with acute walt depletion. Arch Dis Child 1956:31: 28-30.

12. Arvanitakis S.N., Loheck C.C.: Metabolic alkalosis and salt depletion in cystic fibrosis. J Pediatr 1973; 82: $535-545$.

13. Fernández $R$., Rodriguez $J$., Barrera $F$, weinberger J.: Diagnóstico del síndrome de Bartler por estudio sistemático de la hipokalemia. Rev Chl Pediatr 1982: $53: 229-233$.

14. Table S. Guiraldes E., Jarpo S. y col.: Determinación de elctrólitos del sudor en el diagnóstico de fibrosis quística. Lixperiencia de 23 años. Rev Med Chile 1985; 113: 758-762.

15. Orenstein $D$.: Diagnosis of cystic fibrosis. Seminars in Respiratory Medicine 1985; 6: 252-260.

16. Mace J., Shanberger J.: Elevated sweat chlorides in a clila with malnutrition. Clin Pediatr 1971: 10 285-286. 